\title{
International Society of Managing and Technical Editors' Asian Conference 2016
}

\author{
Hyun Jung Yi \\ Medical Library, Hanyang University Guri Hospital, Guri, Korea
}

The International Society of Managing and Technical Editors (ISMTE) is an organization that was established in 2007 serving working editors. While the ISMTE annual conference is usually held in the United States or Europe, the 2016 conference was held in Asia for the first time, from April 4th to the 5th at Singapore Clarke Quay Hotel. World-renowned publishers such as Wiley, American Chemical Society Publications, American Institute of Physics, Nature Publishing Group, Wolters Kluwer, Oxford University Press, Elsevier, Taylor \& Francis, Thomson Reuters, etc. are registered as corporate members of ISMTE. At this year's conference, publishers and their affiliates such as Editage, Aries systems, Cabell's International, Council of Science Editors, ANSInet, etc. participated as sponsors. Over 100 individuals from all over the world attended the conference in order to improve the quality of academic publishing. The participants were editors belonging to companies that run editing or publishing related businesses, and editors belonging to academic societies or journal editorial boards.

There were a total of 12 sessions during the two-day conference. The first session on April 4th dealt with peer reviews, where lectures titled, "Maintaining publication ethics at scale," "Peer review present and future: ethical issues and challenges", and "Finding value in traditional peer review" were presented. A chairperson introduced the speakers for each of the talks. Representatives from the PLoS (Public Library of Science), the Committee on Publication Ethics (COPE), and American Chemical Society spoke of the need to carry out peer reviews with shared responsibility between authors, reviewers, readers, and academic communities. Initially I was a bit embarrassed when one of the COPE speakers introduced a Korean researcher as an example of a "fake reviewer," but then realized that bringing more attention to this kind of situation might help establish a more reliable peer review system in Korea.

After skimming through the conference schedule, I was most looking forward to the third session. Dr. Jason J. Robert presented a talk titled, "Peer review and editorial office data: measuring and reporting your performance." The speaker introduction mentioned that Dr. Robert

Received: July 6, 2016

Accepted: August 2, 2016

Correspondence to Hyun Jung Yi hjyi@hmc.hanyang.ac.kr

ORCID

Hyun Jung Yi

http://orcid.org/0000-0003-0663-4373 currently works at Original Editorial and had previously been a managing editor at Blackwell Publishing. He has mostly edited American medical journals. Moreover, he was the former president of ISMTE and had participated in editing the CONSORT (Consolidated Standards of Reporting Trials), a guide to randomized controlled trials. His presentation consisted of monitored data on how a manuscript develops through peer reviews. The issues he dealt with were all things I had also thought about while managing an editorial committee. For instance, 
time management is an important part of keeping an editorial committee running smoothly. Dr. Robert presented timetables for manuscript management and data for setting deadlines, etc. drawn from simple surveys targeting peer reviewers. Usually, 14 days are given for evaluation, and $76 \%$ of reviews are completed in this time frame. A reminder is useful in making sure the evaluations are turned in on time, and data shows a reminder letter delivered seven days before the deadline is the most effective practice. Submission management systems could be altered based on these findings. Dr. Robert plans to conduct an analysis on which reviewers have made helpful comments. Editors must make sure that after a manuscript is submitted, time is not wasted. For example, letting a two-week period pass without selecting peer reviewers would be a waste of time. Although many of the submission management systems used in Korea and elsewhere are based on such findings, I think we should come up with more specific improvements related to better time management.

A buffet-style lunch was served. The tables in the restaurant were labeled with a variety of different topics, allowing participants with similar interests to sit together and converse. This arrangement showed the attentiveness of the organizers.

After lunch, I wandered the halls to look at posters because there wasn't any free time set aside for browsing. There wasn't a separate booth for them; they were exhibited along the walls of the lecture hall. A total of 16 were displayed, but because the break was too short, those wanting to read each one of them or speak with the poster presenters may have felt rushed.

In the afternoon session, two talks on "Metrics: tools for discovering best practices" and four talks on "Open access: navigating the changing landscape" were presented. The speakers presented cases that evaluated the influence of a dissertation using emerging methods such as Altmetrics. Andy Nobes, who is part of a non-profit organization called International Network for the Availability of Scientific Papers which supports research and publication of studies by researchers from developing countries, presented his findings on the current state of open access and the research process in member countries. Audience members asked a variety of questions regarding institution repositories and resources for searching for relevant literature. One asked the speaker to rank the most important factors to consider when searching for references. The speaker replied that the order should be: the relevance to one's discipline, journal impact factor, the reputation of the journal, whether the journal is indexed, the quality of the peer review, the relationship of the journal, whether the journal is open access, and the country the journal was published in. Many audience members were not familiar with Creative Commons or data sharing. The speaker announced the number of open access journals in South
America, India, China, the Philippines, and Vietnam and the names of their indexes. When I asked him why Korean journals weren't included, he said he didn't know where to look for them, which I thought was a poor excuse. I gave him the website addresses to ScienceCentral and Synapse so that he could check the number of Korean journals.

One innovative method that was used to engage participants in this year's conference was the Sli.do tool. The organizers set up a room with a password where participants could enter and freely write down questions during the conference. It was a great way to give and receive feedback, transcending the limits of using a microphone, space, and difference in languages and personalities. The organizers were a bit disappointed that the room was underused, but I believe it could be a useful tool for South Koreans, who aren't used to actively asking questions or expressing their opinions. If possible, I want to utilize this tool at a future conference I organize.

The interests of editors and publishers around the world are changing, and technology is at the forefront of changes to existing perceptions and the nature of academic publishing. In the past when a manuscript had to be printed on paper, there were many limitations regarding the amount of content (e.g., the number of articles in a journal), but with the development of the internet, there is no need for such limitations. Therefore, policies have changed, which in turn have changed the perceptions of editors. On the second day of the conference, talks on "Emerging standards as best practices in scholarly publishing" and "Servicing the journal: breakouts for system managers" reminded us of the efforts to standardize meta-data needed for academic publishing and distribution, using tools such as CrossCheck DOI, FundRef, ORCID, eXtyles, JATS XML, etc. Two submissions systems from Thomson Reuters were introduced, ScholarOne and the Aries System. I also learned how manuscripts are imported into the submission management systems, what mechanism is used to internally handle the article processing charge when the journal is open access, and how to provide editors and reviewers with a more efficient search system.

Small-group panel meetings were also organized at COPE via registration. Speakers and participants discussed real-life cases on publishing ethics, which were submitted beforehand. The panels discussed the same issues on both the first and second day of the conference. I thought that through such panels, in addition to presentations, the organizers diversified the conference and tried to listen to the opinions of the participants. In one such case that was introduced, a paper that was the subject of another author's paper was submitted, so the journal editorial committee rejected the second paper on the grounds of concern for ethics and scientific validity. In 


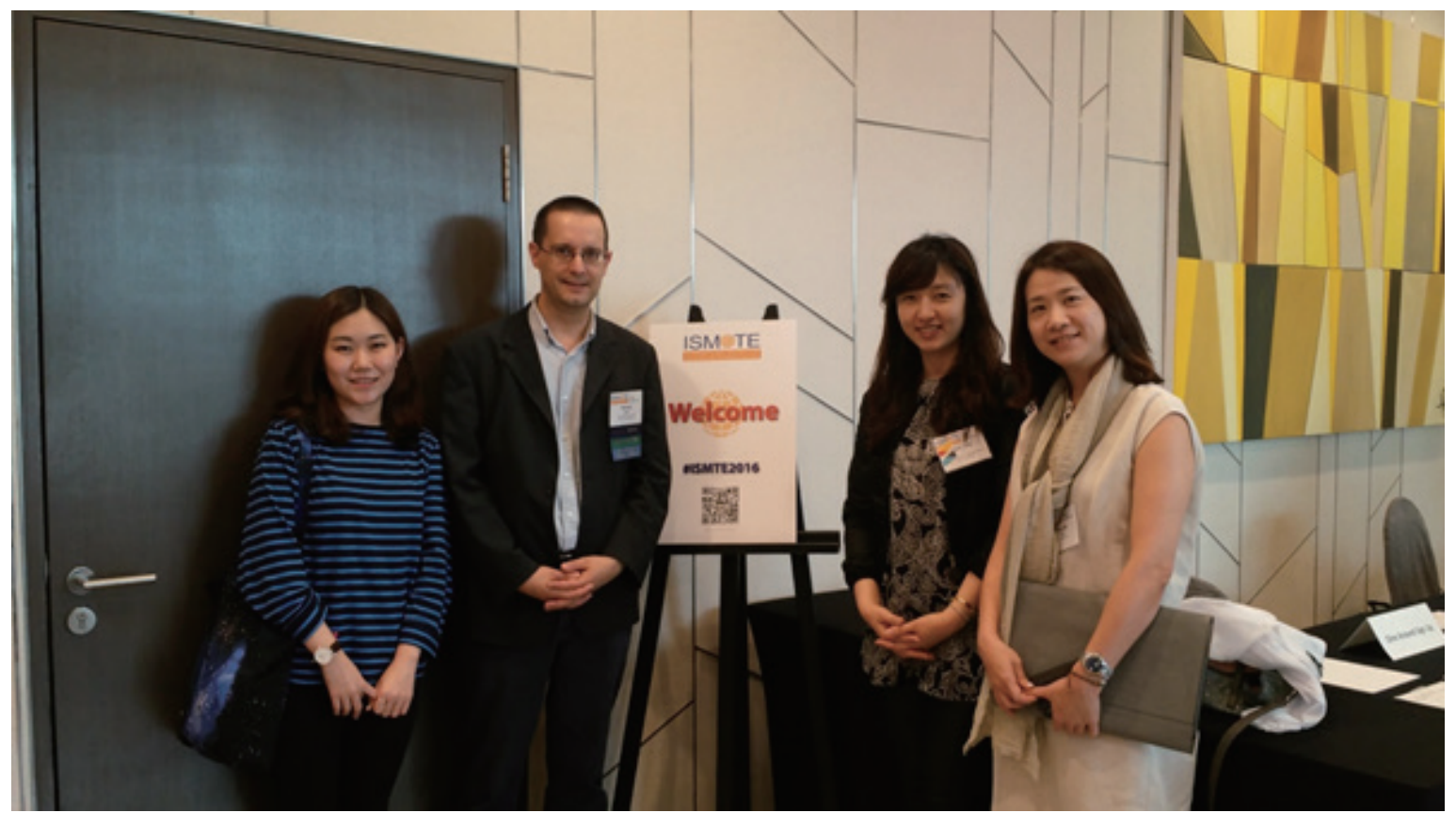

Fig. 1. Korean delegates with the International Society of Managing and Technical Editors President, Michael Willis.

another case, we discussed whether or not authorship could be retracted if a communications writer or academic journal requests confirmation of authorship from the co-author but he or she ignores this request on purpose. While there were no clear-cut solutions to these cases, I realized that there are many issues to think about.

One of the most important issues discussed in academic publishing and editing conferences nowadays is authorship. At ISMTE, many methods to grant author contributions were introduced, such as computation, conceptualization, data curation, data visualization, writing-review, formal analysis, investigation, methodology, project administration, resources, supervision, and testing.

Michael Willis is the chairperson of the ISMTE (Fig. 1). He is part of Wiley, and is the regional manager of the peer review research department. After the photo session, we talked a bit, and it was clear that he was aware of the Council of Asian Science Editors. Through participating in the ISTME this year, I realized that Korean Council of Science Editors and Council of Asian Science Editors workshops and mem- bership community activities are on par with the level of work around the world.

Although there were many different themes presented at the ISMTE, the three most important were peer review, authorship, and Open Access. The conference was a success on many fronts, in its composition of sessions, the content of the presentations, the speakers, the overall organization of the event, etc. I plan to direct my attention to many of the related fields of study. I thank the ISMTE for presenting the opportunity to consider ways to improve an editorial committee.

\section{Conflict of Interest}

No potential conflict of interest relevant to this article was reported.

\section{Acknowledgments}

This work was supported by a travel grant from the Korean Council of Science Editors (2016). 